\title{
Impact of the quality of pastoral care on the incidence of eating disorder symptoms in schools
}

\begin{abstract}
Peak age for eating disorder (ED) onset is 10-19years; therefore schools are well-positioned to promote prevention and support at-risk individuals. However, to date, little is known about the possible role that school-based pastoral support might provide in this context. This study aimed to investigate whether students' ED pathology differed depending on the quality of school-based pastoral care. Four hundred and twenty-five participants from five UK schools ( $52 \%$ female; $\mathrm{n}=221)$ aged $16-19(\mathrm{M}=17.14, \mathrm{SD}=0.76)$ completed the Eating Disorder Examination Questionnaire and the Health of the Nations Outcomes Scale for Children and Adolescents. A rating system was applied to rate each school's pastoral care quality, operationalised as available student support, staff training/knowledge regarding EDs and ED-related school policies. Pastoral care quality had a significant, medium-sized impact on ED symptom prevalence $\left(\eta p^{2}=.06\right)$, with higher quality care resulting in lower ED symptomatology. Additionally, overall wellbeing was also higher in schools with better quality pastoral care $\left(\eta p^{2}=0.05\right)$. High quality pastoral care may be a useful tool in fighting disordered eating in adolescents.
\end{abstract}

Keywords: eating disorders, anorexia nervosa, bulimia nervosa, adolescents, schools, prevention, detection
Volume 7 Issue 4 - 2017

\author{
Amy Harrison, Stephanie Victoria Watterson \\ Regent's School of Psychotherapy \& Psychology, Faculty of \\ Humanities, Regent's University London, UK
}

Correspondence: Amy Harrison, University College London, Department of Psychology and Human Development, Institute of Education, 25 Woburn Square, London, Email,Harrison@ucl.ac.uk

Received: November 29, 2016 | Published: March 16, 2017
Abbreviations: ED, eating disorders; AN, anorexia nervosa; $\mathrm{BN}$, bulimia nervosa

\section{Introduction}

Eating disorders (EDs) including anorexia nervosa (AN) and bulimia nervosa $(\mathrm{BN})$ have been described as one of the most difficult psychiatric disorders to treat, ${ }^{1}$ have a mean illness duration of 7 years ${ }^{2}$ and early intervention during their typical adolescent onset ${ }^{3}$ is associated with better prognosis. ${ }^{4}$ Hospitalisation for EDs in young people has increased in the UK and the illness is recognised as a significant challenge for citizens of the European Union. ${ }^{5}$ In keeping with this, EDs have begun to be recognised in government initiatives regarding wellbeing in young people. ${ }^{6}$ In a systemic model, alongside clinical interventions involving families, other components of the ecological system, including educational settings, could play a vital role in detecting symptoms and supporting recovery, particularly given the portion of time adolescents spend in school. Thus, schools may be an important part of a multidisciplinary network of people, institutions and services well-placed to help limit the risk of EDs developing and/ or becoming enduring illnesses which last into adulthood.

However, to date, little published evidence is available as to the nature and efficacy of schools as a resource. What is known from a previous study which conducted focus groups in 29 UK schools, including 63 staff members, is that although teaching and pastoral staff were aware of EDs amongst their student population, they reported lacking knowledge regarding how best to help at all stages of the illness. ${ }^{7}$ In response to this, a range of model policies, skillsbased resources and training packages were developed, focused on improving understanding around EDs in schools and offering schoolwide policies to support students affected by EDs and developing skills to support recovery. ${ }^{8}$ Before further developing and examining the possible long-term impacts of this package of support, it is however important to better understand whether school-based pastoral care itself in the context of EDs might contribute to reduced rates of symptoms in young people. A number of definitions of pastoral care exist, and there is conflict in the literature regarding the best definition, but one possible conceptualisation is that pastoral care is the business of all school staff members, and relates to any measures designed to assist a young person to reach their full potential, success and happiness. ${ }^{9}$ This is likely to include knowledge, support and school policies around physical and mental wellbeing.

Therefore, this study aimed to explore whether higher pastoral care provision, encompassing knowledge training, policies and school based support, was associated with reduced ED symptoms with the objective of better understanding the potential resource of the educational context in the fight against EDs. It was hypothesised that there would be a difference in ED symptom incidence in schools with higher quality pastoral care compared to those providing lower quality pastoral care.

\section{Materials and methods}

\section{Sample and data collection}

UK secondary schools (both private and state-funded) were approached to participate during a six month recruitment period (January-July 2015) through adverts sent to schools and via personal contacts. To participate, male and female full-time students were required to be aged 16-19, able to complete measures written in English and provide informed consent. Participation was voluntary and students were instructed they may withdraw from the study at any point. A coding system was used to allow withdrawal at a later date whilst also protecting confidentiality. Participants were asked to complete the paper-based questionnaires anonymously in their own time and return them to the researcher. A full written debrief was offered with details of services to contact should participants require further support. The study was approved by the Regent's University London Research Ethics Committee reference 15.09 and the research was conducted in accordance with the Code of Ethics of the World Medical Association Declaration of Helsinki. 
Table I Overview of schools, pastoral care provision and ratings

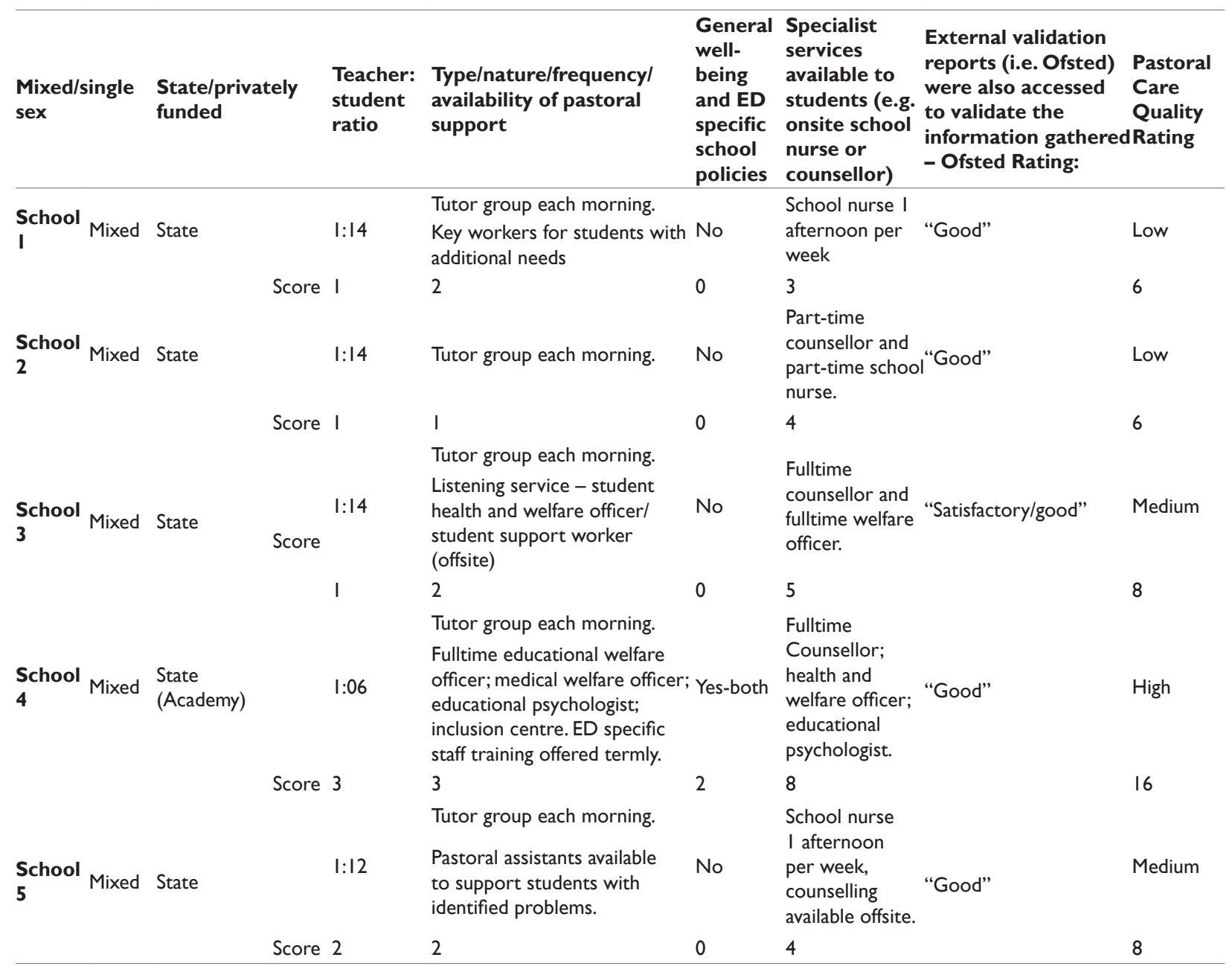

Score refers to the score achieved (out of 4) across the four domains of pastoral care quality, with higher scores indicating higher pastoral care quality. The categorical score is derived from the sum of these domain scores, with schools' rated as "low" scoring $\leq 6$, schools rated as "medium" scoring 7 - I0, schools rated as "high" scoring $>10$.

Table 2 Outcome Data for the Eating Disorders Examination Questionnaire and General Wellbeing across the Five UK Secondary Schools

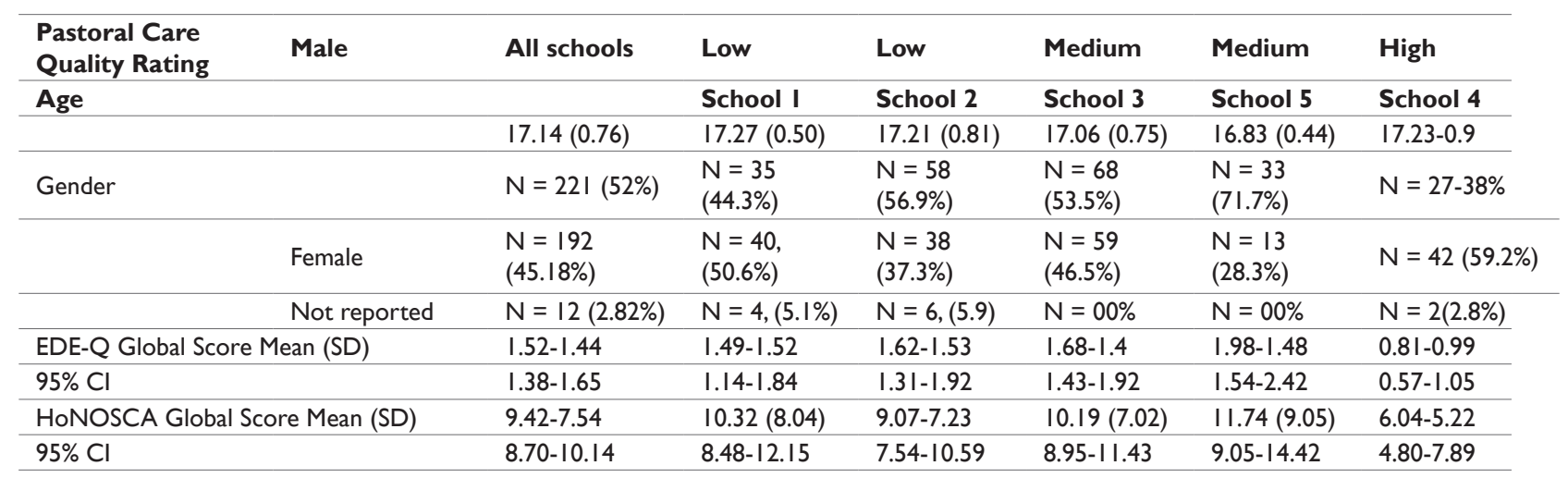

*Percentage (\%) of students was provided based on total number of participants who had provided their weight and height. **Weight for Height Percentile. HoNOSCA $=$ Health of the Nation Outcomes Child and Adolescent Mental Health scale. EDEQ=Eating Disorders Examination Questionnaire. SD=standard deviation.

\section{Measures}

Participants were asked to provide demographic data regarding their gender, age and weight/height which were used to calculate weight for height percentages. ${ }^{10}$
Symptom incidence: ED symptoms were assessed using the 28item Eating Disorder Examination Questionnaire (EDE-Q), ${ }^{11}$ a self-report measure which assesses core attitudinal and behavioural features of EDs, including restraint; eating concern; weight concern; and body shape concern over the preceding 28days. Higher scores 
indicate greater symptoms and the global EDE-Q score was used as the primary outcome measure. Cronbach's alpha in this study was 0.89 , comparable to published data (0.81 to 0.94$).{ }^{12}$ The ED behaviour counts within the measure were used to explore probable ED diagnoses. A cut-off of $>4$ has been used to define ED pathology in community populations. ${ }^{13}$ General psychological wellbeing was assessed using Section A of the Health of the Nation Outcome Scales for Children and Adolescents (HoNOSCA), ${ }^{14}$ a 13-item self-report scale which addresses disruptive/aggressive behaviours, over-activity/ concentration problems, self-injury, substance misuse, academic skills, physical illness, hallucinations/delusions, nonorganic somatic symptoms, emotional symptoms, peer relationships, self-care, family relationships, and school attendance, with higher scores indicating poorer psychological wellbeing. Cronbach's alpha is reported at $0.56^{15}$ and in the current study was 0.89 . The outcome variable used was the global score and this general wellbeing measure was included to explore the specificity of any potential findings regarding pastoral care and ED symptoms.

Quality of pastoral care: After consulting educational leaders, four key domains were identified across which the quality of pastoral care was rated. These domains reflected two key areas:

I. Support available to students; and

II. The level of knowledge and training around EDs for staff. For brevity, the overall quality level is referred to throughout as 'pastoral care quality.' To rate schools' pastoral care quality, schools were asked to provide information across four domains:

a. the type/nature/frequency and availability of pastoral support;

b. general wellbeing and ED specific school policies;

c. specialist services available to students (e.g. onsite school nurse or counsellor) and

d. ED focused staff-training. External validation reports from the Office for Standards in Education, Children's Services and Skills (Ofsted) were also accessed to validate information gathered. A 4-point scale was used to quantify the level of pastoral care quality across all four domains: 1=Outstanding; i.e. reflecting pastoral care quality significantly exceeding statutory requirements and good practice policies; $2=\mathrm{Good}$; i.e reflecting pastoral care quality above statutory requirements and good practice policies; 3=Satisfactory; i.e reflecting pastoral care quality meeting statutory requirements and good practice policies and 4=Inadequate; i.e reflecting pastoral care quality below statutory requirements and good practice policies). Schools' rated as "low" scored $\leq 6$ : meeting statutory requirements, those exceeding the requirements were rated as "medium", scoring 7-10, and if heavily exceeding the requirements, scoring $>10$, pastoral care quality was rated "high." Schools were assigned pseudonyms (15) to protect anonymity.

\section{Data analysis}

Data were analysed using the Statistical Package for the Social Sciences (Version 22) for Windows ${ }^{16}$ and planned analysis was an ANOVA followed by Bonferroni-corrected post-hoc tests. Partial etasquared $\left(\eta p^{2}\right)$ was calculated asan effect size estimate with a small effect corresponding to 0.01 , a medium effect corresponding to 0.06 and a large effect corresponding to $0.14 .{ }^{17}$ Parametric tests were selected after histograms and a Kolmogorov-Smirnov test confirmed data were normally distributed.

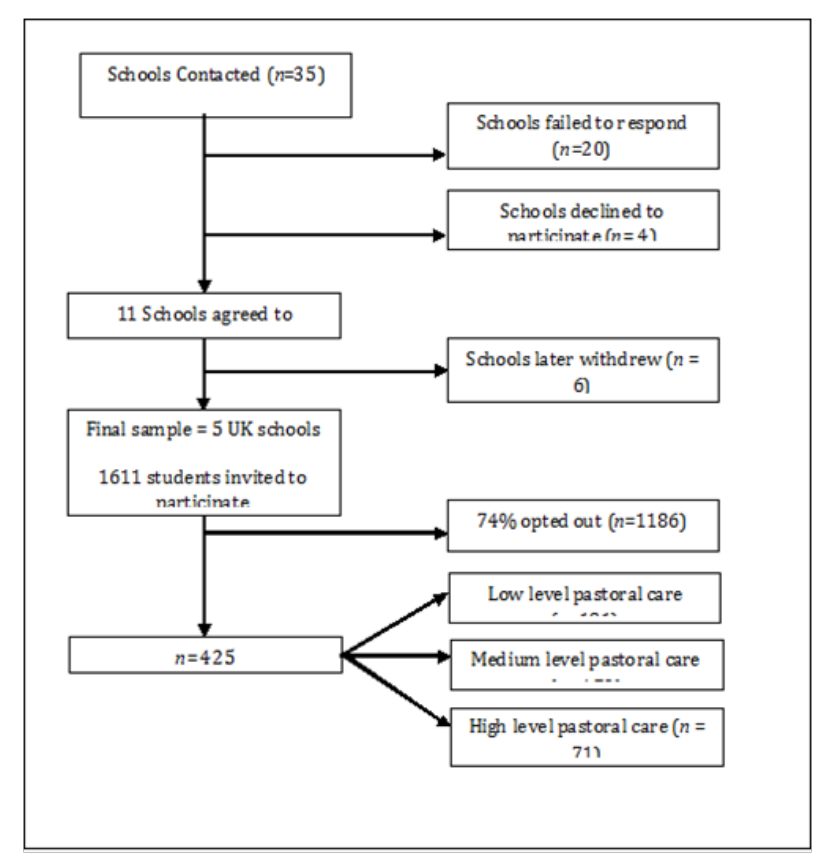

Figure I Recruitment Flowchart.

\section{Results and Discussion}

Initial contact was established with 35 schools who responded to the recruitment materials ( $\mathrm{n}=33$ state-funded; $\mathrm{n}=2$ privately funded) and having been contacted to provide further information, 20 schools ceased correspondence; 4 schools declined to participate and of the 11 remaining schools, six who initially agreed to participate later withdrew before the data collection stage (Figure 1). These schools all withdrew because of a belief that discussing EDs might somehow evoke symptoms in the student population, with common reasoning including: "The head teacher is very protective of the students and is bothered by the anorexia questions... to take part would be going against the school's policy to protect the students; "alongside concerns that their students were "currently battling eating disorders and are in quite a bad way, " with teachers concerned about the "effect the survey will have on the other students and how it will be received by parents."

Following further reassurances regarding confidentiality and anonymity, 5 state-funded schools agreed to allow access to their student population. This take-up rate represents $14.29 \%$ of the schools initially contacted. School 4, was coded as having "high" level pastoral care, schools 3 and 5 were rated "medium" and schools 1 and 2 were coded as offering "low" level pastoral care quality (Table 1).

The final sample of five UK secondary schools had on roll 1611 possible participants who met inclusion criteria and $26 \%(\mathrm{n}=425)$ volunteered to participate. One hundred and ninety-two participants were male (45\%), 221 were female $(52 \%)$ and $3 \%$ of participants did not disclose their gender $(n=12)$. The mean age of the sample was 17.14 ( $\mathrm{SD}=0.76)$. There was a significant, medium-sized difference in the age of participants between schools $(\mathrm{F}(4,411)=3.62, \mathrm{p}=<0.01$, $\left.\eta p^{2}=0.03\right)$. Bonferroni corrected $(0.05 / 12=0.004)$ post-hoc analyses indicated that participants in school 5 were younger than students from schools $1(\mathrm{t}(117.86)=-4.59, \mathrm{p}=<0.001), 2(\mathrm{t}(139.60)=-3.80$, $\mathrm{p}=<0.001)$, and $4(\mathrm{t}(106.26)=-3.20, \mathrm{p}=0.002)$. Gender was not equally distributed between schools $\left(\mathrm{F}(4,408)=3.88, \mathrm{p}=<0.01, \eta \mathrm{p}^{2}=0.04\right)$. 
Bonferroni post-hoc analyses indicated that there were significantly more male participants in school 4 than in school $5(\mathrm{t}(3.64)=101.55$, $\mathrm{p}=<0.001$ ). Given these differences in age and gender across the five participating sites, these variables were controlled for in the analyses.

Across the sample, the mean weight of male participants was $69.06 \mathrm{~kg}(\mathrm{SD}=13.01)$ and it was $58.09 \mathrm{~kg}(\mathrm{SD}=11.05)$ for female participants. Overall, female students reported significantly greater ED symptomatology than males $(\mathrm{M}=2.19, \mathrm{SD}=1.48)$ and lower general wellbeing $(\mathrm{M}=10.62, \mathrm{SD}=8.42)$ than males $(\mathrm{M}=0.79$, $\mathrm{SD}=0.97 ; \mathrm{M}=8.24, \mathrm{SD}=6.31), \mathrm{t}(382.73)=-11.48, \mathrm{p}=0.001 ; \mathrm{t}(396.84)=-$ $3.25, \mathrm{p}=<0.001)$ (Table 2).

There was a significant, medium-sized effect of school setting on the incidence of $\mathrm{ED}$ symptoms $\left(\mathrm{F}(4,420)=6.51, \mathrm{p}<0.01, \eta \mathrm{p}^{2}=0.06\right)$. Bonferroni corrected post-hoc analyses indicated that ED symptoms were significantly lower in school 4 (rated as having high quality pastoral care) compared to school 1(low quality pastoral care) $(\mathrm{t}(135.62)=-3.43, \mathrm{p}=0.001)$, school 2(low quality pastoral care) $(\mathrm{t}(169.95)=-4.36, \mathrm{p}=<0.001)$, school $3(\mathrm{t}(186.90)=5.23, \mathrm{p}=<0.001)$ and school 5 (medium quality pastoral care) $(\mathrm{t}(70.96)=4.76$, $\mathrm{p}=<0.001)$. These effects remained after the potential confounds of age: $F(4,410)=6.02, p=<0.001, \eta p^{2}=0.06$ and gender: $F(4,407)=3.95$, $\mathrm{p}=0.004, \eta \mathrm{p}^{2}=0.05$ were controlled for.

\section{Likely cases of eating disorders across the schools}

After selecting those scoring over the community cut-off (Carter et al., 2001) for the EDEQ, with the aid of diagnostic syntax, ${ }^{18}$ data were examined to investigate the percentage of participants reporting symptoms indicating a likely DSM-5 ${ }^{19}$ diagnosis of AN or BN. Overall, there were $13(3.06 \% ; 10$ females and 3 males) likely cases of AN and 64 (15.06\%; 53 females and 11 males) likely cases of BN.

There were significant differences in the number of likely cases of $\mathrm{AN} ;\left(\mathrm{F}(4,384)=3.14, \mathrm{p}=0.015, \eta \mathrm{p}^{2}=0.03\right)$ depending on pastoral care quality. School 2 (interestingly, a low quality pastoral care setting) and school 4 (a high quality pastoral care setting) had no likely cases of AN. However $2.86 \%(n=2)$ of students in school 1 (low quality pastoral care), $7.56 \%(\mathrm{n}=9)$ of students in school 3 (medium quality pastoral care) and $2.56 \%(\mathrm{n}=2)$ of students in school 5 (medium quality pastoral care) provided data indicating a possible AN diagnosis.

Regarding likely cases of BN, there was no significant main effect of school $\left(\mathrm{F}(4,400)=1.18, \mathrm{p}=0.319, \eta \mathrm{p}^{2}=0.01\right)$. However, there was a trend towards higher rates in lower quality pastoral care settings. School 4 (high quality pastoral care) had the lowest incidence of likely BN, with 6 probable cases $(8.70 \%)$. Conversely, $19(20 \%)$ students from school 2 (a lower quality pastoral care setting) and 13 $(17.33 \%)$ students from school 1 (also a lower quality pastoral care setting) reported symptom severity indicative of a probable diagnosis of BN. Regarding the two schools with medium quality pastoral care, 21 students from school 3 (20.59\%) and 5 from school $5(11.63 \%)$ were likely cases of $\mathrm{BN}$.

There was also a significant, small-sized difference in students' general psychological wellbeing, measured by the HoNOSCA, between schools $\left(\mathrm{F}(4,420)=5.68, \mathrm{p}=<0.001, \eta \mathrm{p}^{2}=0.05\right)$. Bonferroni corrected $(0.05 / 12=0.004)$ post-hoc analyses indicated that participants' overall psychological wellbeing was significantly higher in school 4 (high quality pastoral care) compared to school 1 (low quality pastoral care) $(\mathrm{t}(3.84)=129.85, \mathrm{p}=<0.001)$, school 3 (medium quality pastoral care) $(\mathrm{t}(4.69)=178.05, \mathrm{p}=<0.001)$ andschool 5 (medium quality pastoral care) $(\mathrm{t}(3.87)=64.83, \mathrm{p}=<0.001)$.

\section{Conclusion}

This study aimed to investigate incidence rates of ED pathology across differing levels of pastoral care quality, encompassing support available to students, training and knowledge of staff members in relation to EDs and school policies around EDs. The five schools $(n=425)$ who consented to take part were rated as offering high (school 4), medium (schools 3 and 5) and low (schools 1 and 2) quality pastoral care. The hypothesis, which was that ED symptom incidence rates would differ depending on pastoral care quality, was supported by the data, as schools with higher quality pastoral care had significantly lower ED symptom incidence rates, with a medium effect size and the positive impact of pastoral care quality was also generalised to broader psychological wellbeing. There was also a trend towards there being fewer probable diagnoses of EDs in the higher quality pastoral care settings and higher quality pastoral care was associated with a small-sized reduction in general symptoms of mental illness.

The data highlight that schools with higher, compared to lower, quality pastoral care had a lower incidence of ED symptoms. This provides a clear message to the UK government that investment in ED training and knowledge for education professionals and a greater emphasis on student support around EDscould be vital tools in the costly battle ${ }^{20}$ against sub-clinical and clinically diagnosable EDs in adolescents. Sadly, despite increases in incidence, ${ }^{21}$ EDs are notably absent from European Union HORIZEN 2020 funding streams ${ }^{5}$ and an implication of these findings is that funding large clinical research initiatives which involve the whole system, including the resource of school-based pastoral care, are needed to reduce the onset and impact of these life-threatening disorders during adolescence.

The likely incidence rates of diagnosable EDs across the schools of $3.06 \%$ for AN and $15.06 \%$ for $\mathrm{BN}$ are somewhat higher than reported in reviews of community-based 1 year prevalence studies (e.g. 370 cases per 100,000 for $\mathrm{AN}$ and 1000 cases per 100,000 for $\mathrm{BN}){ }^{22}$ However, this was a volunteer sample with participants at the peakage of onset for both disorders and the anonymous self- reporting may also have influenced symptom disclosure. Indeed, the self-report nature of the measure is a clear limitation of this study and follow-up clinical interviews such as the Eating Disorder Examination ${ }^{23}$ would be required to confirm these likely diagnoses. Although the EDEQ is thought to have strong discriminant validity ${ }^{24}$ and was selected due to its brief nature as a means of reducing participant burden, future studies may wish to consider using other screening tools such as the Eating Disorder Inventory. ${ }^{25}$ The study included a narrow age range and future work may benefit from broadening recruitment to a younger age range.

It is possible that the impact of pastoral care quality of symptoms was a cohort effect influenced by school setting and randomly allocating students to higher or lower pastoral care quality settings across sites would be required to adequately explore this. Furthermore, this study did not explore how pastoral care quality might impact on symptom incidence and whether factors in addition to pastoral care quality may also have played a role. It may be that the schools with higher quality pastoral care were better able to detect possible cases and support families to access effective treatments, thus reducing the incidence in the school population. This aligns with NHS England's guidance which highlights how UK schools should be part of the treatment referral process. ${ }^{26}$ Given that teachers have previously reported a lack of knowledge and skills around supporting students with Eds, ${ }^{7}$ these data further support the need for greater investment 
in nationwide teacher training to improve practice and harness the strengths of schools and their staff to reduce the incidence of ED symptoms in schools.

In conclusion, it is possible that high quality pastoral care may play a role in reducing ED symptom incidence and future studies are needed to further explore the mechanism through which this effect might be found.

\section{Acknowledgements}

The authors would like to thank all schools that took part in the study.

\section{Conflicts of interest}

None to declare.

\section{Funding}

None.

\section{References}

1. Halmi KA, Agras W, Crow S, et al. Predictors of treatment acceptance and completion in anorexia nervosa: implications for future study designs. Arch Gen Psychiatry. 2005;62(7):776-781.

2. Wentz E, Gillberg IC, Anckarsäter H, et al. Adolescent-onset anorexia nervosa: 18-year outcome. Brit JPsychiat . 2009;194(2):168-174.

3. Currin L, Schmidt U, Treasure J, et al. Time trends in eating disorder incidence. Brit J Psychiat. 2005;186:132-135.

4. Lock J, Le Grange D, Agras WS, et al. Treatment Manual for Anorexia Nervosa: A Family-Based Approach. Guildford Press. 2001.

5. Schmidt U, Adan R, Böhm I, et al. Eating Disorders: The Big Issue Lancet Psychiat. 2016;3(4):313-315.

6. Department for Children, Schools and Families (DCSF). Social and Emotional Aspects of Learning (SEAL) Programme: Guidance for Secondary Schools. London UK DCSF. 2007.

7. Knightsmith P, Treasure J, Schmidt U. Spotting and supporting eating disorders in school: recommendations from school staff. Health Edu Res. 2013;28(6):1004-1013.

8. Knightsmith P. Self-Harm and Eating Disorders in Schools: A Guide to Whole-School Strategies and Practical Support. Jessica Kingsley Publishers London UK. 2015.

9. Grove M. The three R's of pastoral care: Relationships, respect and responsibility. Past Care Edu. 2004;22(2):34-38.

10. Golden NH, Yang W, Jacobson MS, et al. Expected body weight in adolescents: comparison between weight-for-stature and BMI methods. Pediatr. 2012;130(6):e1607-e1613.
11. Fairburn CG, Beglin SJ. Assessment of eating disorders: Interview or self-report questionnaire? Int J Eat Disord. 1994;16(4):363-370.

12. Mond JM, Hay PJ, Rodgers B, et al. Validity of the Eating Disorder Examination Questionnaire (EDE-Q) in screening for eating disorders in community samples. Behaviour Research and Therapy. 2004;42(5):551-567.

13. Carter JC, Stewart DA, Fairburn CG. Eating disorder examination questionnaire: norms for young adolescent girls. Behav Res Ther . 2001;39(5):625-632.

14. Gowers SG, Harrington RC, Whitton A, et al. Brief scale for measuring the outcomes of emotional and behavioural disorders in children. Health of the nation outcome scales for children and adolescents (HoNOSCA). Brit JPsychiat . 1999;17:413-416.

15. Tiffin PA, Rolling K. Structure of the Health of the Nation Outcome Scales for Children and Adolescents: An ordinal factor analysis of clinician ratings of a sample of young people referred to community mental health services. Psychiat Res. 2012;197(1-2):154-162.

16. IBM Corporation. IBM SPSS Statistics for Windows, Version 22.0. IBM Corporation. 2013.

17. Cohen JW. Statistical Power Analysis for the Behavioural Sciences. (2nd Edn). Hillsdal, Lawrence Earlbaum Associates, New Jersey, USA. 1988.

18. Mancuso SG, Newton JR, Bosanac P, et al. Classification of eating disorders: comparison of relative prevalence rates using DSM-IV and DSM-5 criteria. Brit J Psychiat. 2015;206(6):519-220.

19. American Psychiatric Association.Diagnostic and Statistical Manual of Mental Disorders, (5th Edn). Washington DC: American Psychiatric Publishing, USA. 2013.

20. Gustavsson A, Svensson M, Jacobi F, et al. Cost of disorders of the brain in Europe 2010. EurNeuropsychopharm. 2010;21(10):718-779.

21. Micali N, Hagberg KW, PetersenI, et al. The incidence of eating disorders in the UK in 2000- 2009: Findings from the general practice research database. BMJ Open, 3, e002646. 2013.

22. Smink FRE, van Hoeken D, Hoek, HW. Epidemiology of Eating Disorders: Incidence, Prevalence and Mortality Rates. Curr Psychiat Rep. 2012;14(4):406-414.

23. Fairburn CG, Cooper, Z, O'Connor M. Eating disorder examination. In CG Fairburn (Ed). Cognitive Behavior Therapy and Eating Disorders. Guildford: Guilford Press. 2008. p.265-308.

24. Aardoom JJ, Dingemans AE, SlofOp'tLandt MC, et al. Norms and discriminative validity of the Eating Disorder Examination Questionnaire (EDE-Q). Eat Behav . 2012;13(4):305-309.

25. Garner DM. EDI 3: Eating disorder inventory-3: Professional manual. Psychological Assessment Resources. 2004.

26. NHS England. Access and Waiting Time Standard for Children and Young People with an Eating Disorder Commissioning Guide. National Collaborating Centre for Mental Health, London, UK. 2015. 